CERN-TH/2000-178

hep-th/0006247

\title{
$D$-Branes on ALE Spaces and the ADE Classification of Conformal Field Theories
}

\author{
W. Lerche, C.A. Lütken \\ Theory Division, CERN, Geneva, Switzerland \\ and \\ C. Schweigert \\ LPTHE, Université Paris VI, 4 place Jussieu, F-75252 Paris, Cedex 05, France
}

\begin{abstract}
The spectrum of $D 2$-branes wrapped on an ALE space of general ADE type is determined, by representing them as boundary states of $\mathcal{N}=$ 2 superconformal minimal models. The stable quantum states have RR charges which precisely represent the gauge fields of the corresponding Lie algebra. This provides a simple and direct physical link between the ADE classification of $\mathcal{N}=2$ superconformal field theories, and the corresponding root systems. An affine extension of this structure is also considered, whose boundary states represent the $D 2$-branes plus additional $D 0$-branes.
\end{abstract}

CERN-TH/2000-178

June 2000

$\dagger$ On leave from Dept. of Physics, University of Oslo, N-0316 Oslo, Norway 


\section{Introduction}

After their rôle in "minimal" conformal field theories had been discovered [1], $\mathrm{ADE}^{-}$classifications have surfaced in various manifestations in the physics literature; for a recent review, see [2]. However, what has been lacking for a long time is a deeper insight into how these various manifestations are related to each other. In particular, while the CFT partition functions encode some group theoretical data (most notably the exponents $\eta_{j}$, which label the diagonal terms), more basic group theoretical quantities like root systems could not be identified within the (bulk) conformal field theories. It is, however, an old observation [3] that other basic data, in the form of eigenvectors of the Cartan matrix, play a rôle in constructing boundary states associated with the conformal field theories. This point has been stressed recently again in 4 .

That root systems of ADE type appear indeed more naturally on the boundary rather than in the bulk, can easily be seen as follows. As is well known, the ADE classification of modular invariants of $S U(2)_{k}$ can be directly related to modular invariants of the $\mathcal{N}=2$ superconformal minimal models by representing these in terms of cosets $\left.{ }^{-}\left(S U(2)_{k} \times U(1)\right) / U(1)\right)$. The latter can in turn be formulated [6] as LandauGinzburg models based on superpotentials given by Arnold's simple singularities of ADE type [7]. Geometrically, the middle-dimensional homology of the resolution of these singularities is known to be isomorphic to the corresponding root lattices. More precisely: $H_{*}(M, \mathbb{Z}) \cong \Gamma_{R}^{(A D E)}$, where $M \equiv M^{(A D E)}$ denotes an ALE space which is a non-compact model of an ADE singularity on a compact $K 3$ manifold.

Physically this means that D2-branes wrapped around the vanishing cycles of $M$ carry the RR quantum numbers of the charged gauge fields of the corresponding ADE type [8]. A priori, the wrapped $D$-branes are solitonic objects on which open strings end, but they can be represented as boundary states of the CFT. The boundary states thus probe the homology $H_{*}(M, \mathbb{Z})$ of the ALE space and so exhibit somewhat finer details of the geometry than the bulk chiral ring, which probes $H_{\frac{*}{\partial}}(M, \mathbb{C})$. This then completes the chain of connections between the ADE classification of modular invariants and $D$-brane configurations of ADE type, i.e., root systems.

$\diamond$ As usual, this stands for the simply laced Lie algebras of type $A_{n}, D_{n}, E_{6,7,8}$.

$\ddagger$ Note that one can construct further partition functions from this coset which do not describe the $\mathcal{N}=2$ minimal models 5 .

七 From this point of view, the fact that the exponents $\eta_{j}$ of the Lie algebra appear in the bulk partition function is simply a consequence of the fact that the Ishibashi states that underlie the boundary states are determined by the diagonal terms of the bulk partition function. 
Using boundary Landau-Ginzburg theory [9], this line of thoughts has been used recently [10] to analyze the spectrum of quantum $D$-branes on ALE spaces and Seiberg-Witten curves. However, the discussion there was restricted to gauge symmetries of type $S U(N) \sim A_{N-1}$. The purpose of the present brief note is to extend the computation in a systematic and uniform way to all the simply laced Lie algebras.

A convenient setting of the problem is to consider type IIA strings compactified on ALE spaces (for related works on strings and D-branes on ALE spaces see e.g., [11]). Such string compactifications can be described in conformal field theory by LandauGinzburg superpotentials of the form [12]: $W_{G}\left(z, x_{i}, u_{k}\right)=z^{-h}+P_{G}\left(x_{1}, x_{2}, x_{3}, u_{k}\right)$, where $h=h(G)$ and $P_{G}\left(\cdot, u_{k}\right)$ are the dual Coxeter number and simple singularity, respectively, of the corresponding simply laced Lie algebra $G$ of type ADE. More specifically, we will be here interested in the exactly solvable "Gepner" points in the respective moduli spaces, described by the following superpotentials [12]:

$$
\begin{aligned}
W_{A_{h-1}} & =z^{-h}+x_{1}{ }^{h}+x_{2}{ }^{2}+x_{3}{ }^{2} \\
W_{D_{\frac{h}{2}+1}} & =z^{-h}+x_{1}{ }^{\frac{h}{2}}+x_{1} x_{2}{ }^{2}+x_{3}{ }^{2} \\
W_{E_{6}} & =z^{-12}+x_{1}^{4}+x_{2}{ }^{3}+x_{3}{ }^{2} \\
W_{E_{7}} & =z^{-18}+x_{1}{ }^{3} x_{2}+x_{2}^{3}+x_{3}{ }^{2} \\
W_{E_{8}} & =z^{-30}+x_{1}{ }^{5}+x_{2}{ }^{3}+x_{3}{ }^{2} .
\end{aligned}
$$

These non-compact Landau-Ginzburg theories describe smooth conformal field theories with $\widehat{c}=2$, which can also be represented in terms of coset models based on $\left(\frac{S U(2)_{h-2}}{U(1)} \times \frac{S L(2)_{h+2}}{U(1)}\right) / \mathbb{Z}_{h}$ [12,13, 14]. The non-compact $z$-dependent piece, corresponding to the $S L(2)$ factor in the coset, describes the non-universal degrees of freedom that are not important for our purposes; its rôle is mainly to push the central charge up to the right value, and also to supply a certain contribution to the intersection form that we are going to compute in the present letter.

\section{Boundary state intersection index}

We will now compute the topological intersection index [15, 16] $I_{a, b} \equiv \operatorname{Tr}_{a, b}\left[(-1)^{F}\right]$ of the boundary states associated with the LG theories defined in (1). Since these theories are tensor products of the $\mathcal{N}=2$ minimal models and their non-compact counterparts, we begin by discussing the boundary states 17 of the minimal models based on $\frac{S U(2)_{k}}{U(1)}$, at levels $k=h-2$. They are labelled by $|L, M, S\rangle$, where $L=$ $1, \ldots, r \equiv \operatorname{rank}(G), M=-h+1, \ldots, h(\bmod 2 h)$, and finally $S=-1,0,1,2(\bmod 4)$ 
determines the R- or NS-sectors (there is a selection rule that puts a constraint on the labels $L, M, S$, whose precise form will be discussed later). The boundary states can be expanded into the Ishibashi states |\rangle$\rangle$ as follows [18:

$$
\left.|L, M, S\rangle=\sum_{(\ell, m, s)} \frac{\psi_{L}^{(\ell)}}{\sqrt{S_{(0,0,0)}^{(\ell, m, s)}}} e^{i \frac{\pi}{h}\left(m M-\frac{h}{2} s S\right)}|\ell, m, s\rangle\right\rangle
$$

(up to normalization). Here, the Ishibashi labels $\ell$ run over the exponents $₫ \eta_{j}$ associated with the Lie algebra $G:(\ell+1) \in\left\{\eta_{j}\right\} \equiv \mathcal{E}(G)$ ( $m$ and $s$ run like $M$ and $S$ as explained above), and

$$
S_{(\ell, m, s)}^{\left(\ell^{\prime}, m^{\prime}, s^{\prime}\right)}=\frac{1}{\sqrt{2} h} \sin \left[\frac{\pi}{h}(\ell+1)\left(\ell^{\prime}+1\right)\right] \exp \left[i \frac{\pi}{h}\left(m m^{\prime}-\frac{h}{2} s s^{\prime}\right)\right]
$$

are the modular transformation matrices of the $\mathcal{N}=2$ characters. Moreover, $\psi_{L}^{(\ell)}$ are the orthonormal eigenvectors of the ADE Cartan matrix $\mathcal{C}(G)$, with eigenvalues $\gamma_{\ell} \equiv 2-2 \cos \left[\pi \eta_{\ell} / h\right], \ell=1, \ldots, r$ It was shown in [4] that the analogous boundary states $|L\rangle$ of $\widehat{S U(2)_{k=h-2}}$ indeed solve the Cardy condition 19.

Note that the labels $\ell$ and $L$ are in general on logically different footings: while $(\ell+1)$ labels the exponents $\eta_{j}(G), L$ labels the components of the eigenvectors of $\mathcal{C}(G)$. This is in accordance with what was said in the introduction: namely that the boundary states are naturally associated with the homology lattice, $H_{*}(M, \mathbb{Z})$, which in the present situation is given by the root lattice. On the other hand, the bulk chiral ring, which is isomorphic to the cohomology $\operatorname{ring} H_{\frac{*}{\partial}}(M, \mathbb{C})$, is associated with the exponents of the simple singularity. Only for the $A$ series there is no distinction between the bulk and the boundary fusion algebras, that is, between the indices $L$ and $\ell$ and between $\psi_{L}^{\left(\ell^{\prime}\right)}$ and $S_{\ell}^{\left(\ell^{\prime}\right)}$.

We will now adopt a particular convention for labeling the root system, following [20]. That is, we split the simple roots $\alpha_{i}$ according to a bi-coloration of the Dynkin diagram, so that we obtain two orthogonal subsets of mutually commuting roots. Following the notation of ref. [3], we represent this in the following way: $\left\{\alpha_{i}\right\} \equiv$

$\dagger$ Explicitly, exponents $\eta_{j}$ and Coxeter numbers $h$ are: $\mathcal{E}\left(A_{n}\right)=\{1,2, \ldots, n\}, h\left(A_{n}\right)=n+1$, $\mathcal{E}\left(D_{n}\right)=\{1,3,5, \ldots, 2 n-3, n-1\}, h\left(D_{n}\right)=2 n-2, \mathcal{E}\left(E_{6}\right)=\{1,4,5,7,8,11\}, h\left(E_{6}\right)=12$, $\mathcal{E}\left(E_{7}\right)=\{1,5,7,9,11,13,17\}, h\left(E_{7}\right)=18$, and $\mathcal{E}\left(E_{8}\right)=\{1,7,11,13,17,19,23,29\}, h\left(E_{8}\right)=$ 30 , respectively.

$\diamond$ If $G$ is of type $D_{\text {even }}$ or $E_{6,8}$, these two sets correspond to symmetry preserving and symmetry breaking boundary conditions, as discussed in [21]. 
$\left\{a_{\bullet}\right\} \cup\left\{a_{\circ}\right\}$. Given these two sets of labels, we resolve the sign ambiguities of the eigenvectors as follows:

$$
\psi_{\bullet}^{(\ell)}=\psi_{\bullet}^{(h-\ell)}, \quad \psi_{\circ}^{(\ell)}=-\psi_{\circ}^{(h-\ell)}
$$

The intersection index can now be computed [16,22] by evaluating an overlap amplitude in the $R R$-sector:

$$
I\left(L_{1}, L_{2}, M_{1}, M_{2}, S_{1}, S_{2}\right) \equiv{ }_{\mathrm{RR}}\left\langle L_{1}, M_{1}, S_{1} \mid L_{2}, M_{2}, S_{2}\right\rangle_{\mathrm{RR}}
$$

For fixed $S$ and $L$ labels, this can be viewed as a $2 h \times 2 h$ matrix whose components are labeled by the $M_{i}$. Inserting the expansion (2), restricting to the $R R$ ground states (achieved by setting $m=\ell+1$ and $s=1$ in the sum), remembering that the overlap in the RR sector carries a phase $e^{i \pi Q}=e^{i \frac{\pi}{h}(\ell+1)}$, and moreover suppressing the $S$ labels on the l.h.s, (5) becomes

$$
\left(I_{L_{1}, L_{2}}^{A D E}\right)_{M_{1}}^{M_{2}}=\sum_{\ell+1 \in \mathcal{E}(G)} \frac{\left(\psi_{L_{1}}^{(\ell)}\right)^{*} \psi_{L_{2}}^{(\ell)}}{S_{(0,0,0)}^{(\ell, \ell+1,1)}} e^{i \frac{\pi}{h}(\ell+1)\left(M_{2}-M_{1}+1\right)} e^{-i \frac{\pi}{2}\left(S_{2}-S_{1}\right)}
$$

The exponential involving the $M_{i}$ can be rewritten in terms of a sine function, so that we get:

$$
\begin{aligned}
\left(I_{L_{1}, L_{2}}^{A D E}\right)_{M_{1}}^{M_{2}} & =(-1)^{\left(S_{2}-S_{1}\right) / 2} \sum_{\ell+1 \in \mathcal{E}(G)}\left(\psi_{L_{1}}^{(\ell)}\right)^{*} \psi_{L_{2}}^{(\ell)} S_{M_{2}-M_{1}+1}^{\ell}\left(S_{0}^{\ell}\right)^{-1} \\
& \equiv(-1)^{\left(S_{2}-S_{1}\right) / 2} N_{L_{1}, L_{2}}^{M_{2}-M_{1}}
\end{aligned}
$$

where $S_{\ell}{ }^{\left(\ell^{\prime}\right)}=\frac{1}{\sqrt{2} h} \sin \left[\frac{\pi}{h}(\ell+1)\left(\ell^{\prime}+1\right)\right]$ are the $S$-matrices and $N_{L_{1}, L_{2}}^{\ell}$ nothing but the boundary fusion coefficients of $\widehat{S U(2})_{h-2}$ for the corresponding $A D E$ type modular invariant [4]. The fact that a computation involving an $\mathcal{N}=2$ superconformal minimal model yields the fusion coefficients of an $S U(2) \mathrm{WZW}$ model, is perhaps not too surprising in view of the observation [12] that the $\mathcal{N}=2$ minimal model, when tensored with the non-compact $S L(2)$ theory, turns into an $S U(2)$ WZW model plus some additional free fields.

We are not yet done, because we still need to identify the group theoretical meaning of the intersection index $I_{L_{1}, L_{2}}^{A D E}$. For this we can make use of certain group theoretical facts [23] that involve the Coxeter element $w$ of the Weyl group, $\mathcal{W}(G)$. 
They have been very useful in the past in the context of integrable systems [24, 25, 3], and indeed we will draw on some of the results of these papers.

Recall that the Coxeter element is the unique (up to conjugation) element of the Weyl group that is of order $h=h(G)$ (that's why $h$ is called the Coxeter number). Physically, $\mathbb{Z}_{h}$ is simply the $R$-symmetry of the LG potentials (1). If we denote by $r_{i}$ a Weyl reflection in the simple root $\alpha_{i}$, then a useful representation of the Coxeter generator is given by $w=w_{(\bullet)} \cdot w_{(\circ)} \in \mathbb{Z}_{h} \subset \mathcal{W}(G)$, where

$$
w_{(\bullet)}=\prod r_{\bullet}, \quad w_{(\circ)}=\prod r_{\circ}
$$

The important point for us is that the roots of $G$ decompose into $r=\operatorname{rank}(G)$ orbits of the Coxeter element, which are one-to-one to the nodes of the Dynkin diagram $\Delta(G)$. More specifically, one can identify a unique representative $\phi_{i}, i=1, \ldots, r$ for each orbit, by demanding that if $\phi_{i}$ is a positive root, then $w \cdot \phi_{i}$ is a negative root. Concretely, one can write the following explicit representation [3]:

$$
\phi_{\bullet}=w_{(\circ)} \cdot \alpha_{\bullet}, \quad \phi_{\circ}=\alpha_{\circ}
$$

In this way we have a direct correspondence between the boundary state labels $L_{i}$ and the $\mathbb{Z}_{h}$ orbits of the roots of $G$.

We are now ready to make use of the following formula that was proven in ref. [3]:

$$
N_{L_{1}, L_{2}}^{\ell+u_{1}-u_{2}}=\left\langle\lambda_{L_{1}}, w^{-\ell / 2} \phi_{L_{2}}\right\rangle
$$

where $\lambda_{L}$ is the fundamental weight dual to the simple root $\alpha_{L}$, and $u_{i}=0$ iff $L_{i} \in\left\{L_{\bullet}\right\}$ and $u_{i}=1$ iff $L_{i} \in\left\{L_{\circ}\right\}$. This formula makes the crucial step in translating the ADE boundary fusion coefficients into inner products in the ADE weight space.

In the final step the non-compact piece $z^{-h}$ of the LG tensor products (1) comes into play. As was argued in [10], it contributes a factor $\left(1-w^{-1}\right)$ to the intersection index and this can be used to convert the fundamental weight to the Coxeter orbit representative [23]: $\phi_{L}=\left(1-w^{-1}\right) \cdot \lambda_{L}$. We thus obtain alltogether (suppressing the $S$ labels):

$$
\left(I_{L_{1}, L_{2}}^{A L E}\right)_{M_{1}}^{M_{2}}=\left\langle\phi_{L_{1}}, w^{\left(M_{1}-M_{2}+u_{2}-u_{1}\right) / 2} \phi_{L_{2}}\right\rangle
$$

where $L_{i}=1, \ldots, r$ and $M_{i}=-h+1, \ldots, h$. Note that the $u_{i}$ implement the "grading" induced by the bi-coloration of the Dynkin diagram, which can be expressed in terms of the selection rule: $u_{i}+M_{i}+S_{i}=0 \bmod 2$ (generalizing the selection rule $L_{i}+M_{i}+S_{i}=$ 
$0 \bmod 2$ for the $A$-series). Since (11) is periodic in the $M$ labels, we can rewrite it in terms of the cyclic $2 h \times 2 h$ shift generator $\gamma(2 h)\left(\right.$ with $\left.\gamma(2 h)^{2 h}=1\right)$ as follows:

$$
I_{L_{1}, L_{2}}^{A L E}=\sum_{k=0}^{h-1}\left\langle\phi_{L_{1}}, w^{k} \phi_{L_{2}}\right\rangle \gamma(2 h)^{2 k+u_{2}-u_{1}}
$$

which makes the $\mathbb{Z}_{h}$ symmetry of the potentials (1) manifest.

Eq. (12) is precisely what was expected: the boundary states are one-to-one to the roots of $G$ (organized in orbits of the $\mathbb{Z}_{h}$ Coxeter symmetry labelled by $L_{i}$ ), and moreover their intersection index gives the inner product between root vectors. This shows that the $\mathcal{N}=2$ minimal model boundary states indeed correspond to $D$-branes wrapped around the primitive cycles of the ALE space.

\section{Affine extension including Do-branes}

Note that we have obtained a finite spectrum of BPS states (wrapped D2 branes) on the ALE space, in one-to-one correspondence to the finite number of diagonal primary fields of the ADE type $\mathcal{N}=2$ superconformal minimal models. It is given in terms of the roots of the gauge group, precisely what is expected for the rigid field theory limit of the type IIA string compactification in which we send the string scale to infinity. It is remarkable that the truncation of the CFT fusion rules has precisely the right structure to select within the root lattice the finite root system.

However, at finite string scale we expect infinitely many further BPS states, in particular D0 brane (or Kaluza-Klein) states with arbitrary positive charge $n$. These are expected to extend the finite root system to an affine one [26] (for a recent exposition, see e.g., [27]). In our framework they should arise from the coupling to the non-compact sector of the LG models (1). While at the moment it is unclear to us how this works for general groups, the situation is much simpler for the $A$-series, where there is no distinction between the bulk and boundary labels, $\ell$ and $L$.

That is, the sine functions in $\psi_{L}^{\ell}=S_{L}^{\ell}$ (c.f., eq. (3)) allow for a natural periodic extension of the labels $L$ that appear in the fusion coefficients $N$ and intersection matrices $I$. This allows to formally generate an infinite spectrum of BPS charges, starting from a basic set of "fractional brane" charges, $\vec{q}_{(0)}$. More precisely, by choosing a different Coxeter element than before (namely $w=\prod r_{i}$ ), we can group all the simple roots in the $\ell=0$ orbit, together with the extending root $\alpha_{h}: \vec{q}_{(0)}=\left\{\alpha_{1}, \ldots, \alpha_{h-1}, \alpha_{h}\right\}$; 
this was the choice that was made in ref. [10]. We can then generate further charges by acting with the step generator [28]

$$
t_{\ell}=\sum_{k=-\ell / 2}^{\ell / 2} \gamma(2 h)^{2 k}
$$

in the following way:

$$
\vec{q}_{(\ell)}=\vec{q}_{(0)} \cdot t_{\ell}
$$

As shown in [10], for the standard range $\ell=0, \ldots,(h-2)$, this reproduces all the roots of $A_{h-1}$, with $I_{\ell_{1}, \ell_{2}}=t_{\ell_{1}} \cdot \mathcal{C} \cdot t_{\ell_{2}}{ }^{t}$ as their intersection matrix ( $\mathcal{C}$ denotes the extended Cartan matrix of $A_{h-1}$ ).

Extending now the range to arbitrary $\ell \in \mathbb{Z}_{+}$, we first of all note that there are gaps in the spectrum whenever $\ell=h-1(\bmod h)$, which is where the intersection index vanishes: $I_{h-1(\bmod h), \ell_{2}}=0$. Moreover we find that (up to ordering of the components): $\vec{q}_{(\ell+n h)}=\vec{q}_{(\ell)}+n \delta, n \in \mathbb{Z}_{+}$, where $\delta \equiv \sum_{i=1}^{h} \alpha_{i}$ (this follows from the fact that the highest root $\delta$ is associated with the corner entry of the shift generator). Accordingly, $I_{\ell_{1}+n_{1} h, \ell_{2}+n_{2} h}=I_{\ell_{1}, \ell_{2}}$. This gives the requisite extension of the root system to the one of $\widehat{A}_{h-1}$, obtained by adding to $\left\{\alpha_{i}\right\}$ the imaginary simple root $\delta$, with $\langle\delta, \delta\rangle=0$. More precisely, the BPS spectrum we get in this way corresponds to the positive roots of $\widehat{A}_{h-1}$ :

$$
\left\{\widehat{\alpha}_{+}\right\}=\left\{\alpha_{+}+n \delta, n \geq 0\right\} \cup\left\{-\alpha_{+}+n \delta, n>0\right\} \cup\{n \delta, n>0\},
$$

where $\alpha_{+}$are the positive roots of $A_{h-1}$. Physically, the first set corresponds to the wrapped $D 2$-branes with $\ell=0, \ldots,[k / 2](k \equiv h-2)$ plus their $K K$ excitations; the second set to the branes with $\ell=[k / 2]+1, \ldots, k$, which may be viewed as anti- $D 2$ branes (associated with negative $\ell$ shifted by $h$ ) plus $K K$ excitations. The last set corresponds of course to the pure $K K$ modes, or $D 0$ bound states.

To motivate the above construction, note that the same algebraic structure (a finite spectrum repeated infinitely many times with a null state in between the copies) is familiar from $2 \mathrm{~d}$ topological minimal models coupled to topological gravity. In fact it was argued in [12] that the LG models (11) are closely related to such systems.

More specifically, it is known for $A_{h-1}$ that the ground ring [29] is given by the finite chiral primary ring of the (twisted) $\mathcal{N}=2$ minimal model, times a tower of infinitely many gravitational descendants [30]: $\left\{x^{\ell} y^{n}\right\}, \ell=0, . ., h-2$, and $n \in \mathbb{Z}_{+}$. 
Actually, the extended spectrum can be entirely constructed from within the LG model (so that $\left\{x^{\ell} y^{n}\right\} \cong\left\{x^{\ell+n h}\right\}$ ), by suitably defining physical states in terms of equivariant cohomology [31]. This is the same structure that we seem to find for the boundary states, with the understanding that each ring element leads to a whole $\mathbb{Z}_{h}$ orbit of BPS states.

Our point of view is, therefore, that the infinitely many BPS states (D2-branes wrapped around the cycles of the ALE space plus $n$ D0-branes on top of that) should be nothing but the boundary counterpart of the gravitationally extended chiral ring of the bulk. This observation may be useful for finding a BCFT or boundary LG description of the $D_{0}$ branes on $K 3$.

\section{ALE fibrations and $\mathcal{N}=2$ Yang-Mills theories}

It is straightforward to extend our results to $\mathcal{N}=2 d=4$ supersymmetric gauge theories of general ADE type, and determine their non-perturbative BPS spectra at the origin of the respective moduli spaces. As mentioned in ref. [10], the corresponding LG potentials are obtained by a particular fibration of the ALE spaces over $\mathbb{P}^{1}$, which amounts to setting $z^{-h} \rightarrow z_{1}^{-2 h}+z_{2}^{-2 h}$ in (四). The intersection index of D3-brane boundary states wrapping the compact cycles of the fibered ALE is obtained by including an extra factor of $\left(1-w^{-1}\right)$ in (12), and looks explicitly:

$$
I_{L_{1}, L_{2}}^{S W}\left(G_{h}=A D E, N_{f}=0\right)=\left(1-g^{-1}\right)^{2} \sum_{k=0}^{h-1}\left\langle\lambda_{L_{1}}, w^{k} \phi_{L_{2}}\right\rangle g^{2 k+u_{2}-u_{1}},
$$

with $g=\gamma(4 h)^{2}$. This is now identified with the intersection form of the vanishing cycles of the Seiberg-Witten curves, from which the strong coupling spectrum of dyons at the $\mathbb{Z}_{2 h}$ symmetric point of the moduli space can be extracted, as explained in [10].

The fibration procedure is not unique, and other fibrations of the same ALE space give rise to $\mathcal{N}=2$ gauge theories with matter fields [32]. While most of the possibilities do not lead to tensor product models and thus are hard to deal with, we find that $S U\left(N_{c}\right)$ with $N_{f}=N_{c}-1$ massless matter multiplets is very simply described by:

$$
W_{N_{c}, N_{f}=N_{c}-1}^{S W}=\frac{1}{z_{1} N_{c}+1}+\frac{1}{z_{2} N_{c}\left(N_{c}+1\right)}+x^{N_{c}},
$$

$\dagger$ For related work see also [33. 
whose intersection form is:

$$
I_{L_{1}, L_{2}}^{S W}\left(N_{c}, N_{f}=N_{c}-1\right)=t_{L_{1}}\left(1-g^{-N_{c}}\right)\left(1-g^{-1}\right)\left(1-g^{N_{c}+1}\right) t_{L_{2}}^{T}
$$

with $g=\gamma\left(2 N_{c}\left(N_{c}+1\right)\right)^{2}$. Our boundary CFT methods allow a straightforward determination of the strong coupling dyon spectrum of these gauge theories at the $\mathbb{Z}_{N_{c}\left(N_{c}+1\right)}$ symmetric origin of their moduli spaces.

\section{Acknowledgements}

We like to thank Peter Mayr for discussions, and Cumrun Vafa as well as the referee for comments on the manuscript. 


\section{References}

[1] A. Cappelli, C. Itzykson and J. B. Zuber, Modular Invariant Partition Functions In Two-Dimensions, Nucl. Phys. B280 445 (1987); The ADE Classification Of Minimal And $A_{1}^{(1)}$ Conformal Invariant Theories, Commun. Math. Phys. 1131 (1987).

[2] J. Zuber, CFT, BCFT, ADE and all that, hep-th/0006151.

[3] P. Dorey, Partition functions, intertwiners and the Coxeter element, Int. J. Mod. Phys. A8 193 (1993), hep-th/9205040.

[4] R. E. Behrend, P. A. Pearce, V. B. Petkova and J. Zuber, Boundary conditions in rational conformal field theories, Nucl. Phys. B570 525 (2000), hep-th/9908036.

[5] T. Gannon, $U(1)^{m}$ modular invariants, $N=2$ minimal models, and the quantum Hall effect, Nucl. Phys. B491 659 (1997), hep-th/9608063.

[6] C. Vafa and N. Warner, Catastrophes And The Classification Of Conformal Theories, Phys. Lett. B218 51 (1989);

E. J. Martinec, Criticality, Catastrophes And Compactifications, Print-89-0373 (EFI,CHICAGO), In Brink, L. (ed.) et al.: Physics and mathematics of strings 389-433.

[7] V. Arnold, S. Gusein-Zade and A. Varchenko, Singularities of Differentiable Maps, Birkhäuser, 1988.

[8] C. M. Hull and P. K. Townsend, Unity of superstring dualities, Nucl. Phys. B438 109 (1995), hep-th/9410167; Enhanced gauge symmetries in superstring theories, Nucl. Phys. B451 525 (1995), hep-th/9505073;

E. Witten, Some comments on string dynamics, Contributed to STRINGS 95: Future Perspectives in String Theory, Los Angeles, CA, 13-18 Mar 1995, hepth/9507121.

[9] K. Hori and C. Vafa, Mirror symmetry, hep-th/0002222;

S. Govindarajan and T. Jayaraman, On the Landau-Ginzburg description of boundary CFTs and special Lagrangian submanifolds, hep-th/0003242; K. Hori, A. Iqbal and C. Vafa, D-branes and mirror symmetry, hep-th/0005247.

[10] W. Lerche, On a boundary CFT description of nonperturbative $N=2$ Yang-Mills theory, hep-th/0006100. 
[11] D. Anselmi, M. Billo, P. Fre, L. Girardello and A. Zaffaroni, ALE manifolds and conformal field theories, Int. J. Mod. Phys. A9 3007 (1994), hep-th/9304135;

M. Bershadsky, C. Vafa and V. Sadov, D-Strings on D-Manifolds, Nucl. Phys. B463 398 (1996), hep-th/9510225;

H. Ooguri and C. Vafa, Two-Dimensional Black Hole and Singularities of CY Manifolds, Nucl. Phys. B463 55 (1996), hep-th/9511164;

C. Vafa, Instantons on D-branes, Nucl. Phys. B463 435 (1996), hep-th/9512078;

C. V. Johnson and R. C. Myers, Aspects of type IIB theory on ALE spaces, Phys. Rev. D55 6382 (1997), hep-th/9610140;

M. R. Douglas and G. Moore, D-branes, Quivers, and ALE Instantons, hepth/9603167;

J. A. Harvey and G. Moore, On the algebras of BPS states, Commun. Math. Phys. 197489 (1998), hep-th/9609017;

M. R. Douglas, Enhanced gauge symmetry in M(atrix) theory, JHEP9707 004 (1997), hep-th/9612126;

D. Diaconescu and J. Gomis, Duality in matrix theory and three dimensional mirror symmetry, Nucl. Phys. B517 53 (1998), hep-th/9707019;

T. Takayanagi, String creation and monodromy from fractional $D$-branes on ALE spaces, JHEP0002 040 (2000), hep-th/9912157;

B. Fiol and M. Marino, BPS states and algebras from quivers, hep-th/0006189.

[12] H. Ooguri and C. Vafa, Two-Dimensional Black Hole and Singularities of CY Manifolds, Nucl. Phys. B463 55 (1996), hep-th/9511164.

[13] A. Giveon and D. Kutasov, Little string theory in a double scaling limit, JHEP9910 034 (1999), hep-th/9909110.

[14] T. Eguchi and Y. Sugawara, Modular invariance in superstring on Calabi-Yau n-fold with A-D-E singularity, Nucl. Phys. B577 3 (2000), hep-th/0002100;

S. Mizoguchi, Modular invariant critical superstrings on four-dimensional Minkowski space x two-dimensional black hole, JHEP0004 014 (2000), hep-th/0003053.

[15] M. R. Douglas and B. Fiol, D-branes and discrete torsion. II, hep-th/9903031.

[16] I. Brunner, M.R. Douglas, A. Lawrence and C. Römelsberger, D-branes on the quintic, hep-th/9906200.

[17] See e.g.,: A. Recknagel and V. Schomerus, D-branes in Gepner models, Nucl. Phys. B531 185 (1998), hep-th/9712186; 
J. Fuchs and C. Schweigert, Branes: From free fields to general backgrounds, Nucl. Phys. B530 99 (1998), hep-th/9712257.

[18] M. Naka and M. Nozaki, Boundary states in Gepner models, JHEP0005 027 (2000), hep-th/0001037.

[19] J. L. Cardy, Boundary Conditions, Fusion Rules And The Verlinde Formula, Nucl. Phys. B324 581 (1989).

[20] H. Hiller, Geometry of Coxeter groups, Pitman London 1982;

J. Humphreys, Reflection groups and Coxeter groups, Cambridge University Press 1990.

[21] J. Fuchs and C. Schweigert, Solitonic sectors, alpha-induction and symmetry breaking boundaries, hep-th/0006181.

[22] K. Hori, A. Iqbal and C. Vafa, as cited in [9].

[23] B. Kostant, The principal three-dimensional subgroup and the Betti numbers of a complex Lie group, Am. J. Math. 81 (1959) 973.

[24] W. Lerche and N. P. Warner, Polytopes and solitons in integrable, N=2 supersymmetric Landau-Ginzburg theories, Nucl. Phys. B358 571 (1991).

[25] P. Dorey, Root systems and purely elastic $S$ matrices, Nucl. Phys. B358 654 (1991); Root systems and purely elastic $S$ matrices. 2, Nucl. Phys. B374 741 (1992), hep-th/9110058.

[26] H. Nakajima, Instantons on ALE spaces, quiver varieties, and Kac-Moody algebras, Duke Math. 76 (1994) 365; Gauge theory on resolutions of simple singularities and affine Lie algebras, Int. Math. Res. Not. 2 (1994) 61; Instantons and affine Lie algebras, alg-geom/9502013.

[27] B. Fiol and M. Marino, as cited in [11].

[28] D. Diaconescu and C. Römelsberger, D-branes and bundles on elliptic fibrations, hep-th/9910172.

[29] E. Witten, Ground ring of two-dimensional string theory, Nucl. Phys. B373 187 (1992), hep-th/9108004.

[30] K. Li, Topological gravity with minimal matter, Nucl. Phys. B354 (1991) 711;

S. Govindarajan, T. Jayaraman and V. John, Chiral rings and physical states in $c<1$ string theory, Nucl. Phys. B402 118 (1993), hep-th/9207109; 
[31] A. Losev, Descendants constructed from matter field and K.Saito higher residue pairing in Landau-Ginzburg theories coupled to topological gravity, preprint TPIMINN-92-40-T;

M. Bershadsky, W. Lerche, D. Nemeschansky and N. P. Warner, Extended N=2 superconformal structure of gravity and $W$ gravity coupled to matter, Nucl. Phys. B401 304 (1993), hep-th/9211040.

T. Eguchi, H. Kanno, Y. Yamada and S. Yang, Topological strings, flat coordinates and gravitational descendants, Phys. Lett. B305 235 (1993), hepth/9302048.

[32] S. Katz, A. Klemm and C. Vafa, Geometric engineering of quantum field theories, Nucl. Phys. B497 173 (1997), hep-th/9609239;

S. Katz, P. Mayr and C. Vafa, Mirror symmetry and exact solution of $4 D N=2$ gauge theories. I, Adv. Theor. Math. Phys. 153 (1998), hep-th/9706110.

[33] O. Pelc, Holography, singularities on orbifolds and $4 D N=2 S Q C D$, JHEP0003 012 (2000), hep-th/0001054. 